\title{
Metapopulation Dynamics on Ephemeral Patches
}

\author{
Carolina Reigada, ${ }^{1, \star}$ Sebastian J. Schreiber, ${ }^{2}$ Florian Altermatt, ${ }^{3}$ and Marcel Holyoak ${ }^{4}$
}

1. Department of Entomology and Acarology, Escola Superior de Agricultura Luiz de Queiroz (ESALQ), University of São Paulo, Piracicaba, Brazil; 2. Department of Evolution and Ecology, University of California, Davis, California 95616; 3. Department of Aquatic Ecology, Swiss Federal Institute of Aquatic Science and Technology (Eawag), CH-8600 Duebendorf, Switzerland; and Institute of Evolutionary Biology and Environmental Studies, University of Zurich, Winterthurerstr 190, CH-8057 Zurich, Switzerland; 4. Department of Environmental Science and Policy, University of California, Davis, California 95616

Submitted March 24, 2014; Accepted October 17, 2014; Electronically published January 8, 2015

Online enhancements: appendixes.

\begin{abstract}
A challenge for conservation management is to understand how population and habitat dynamics interact to affect species persistence. In real landscapes, timing and duration of disturbances can vary, and species' responses to habitat changes will depend on how timing of dispersal and reproduction events relate to the landscape temporal structure. For instance, increasing disturbance frequency may promote extinction of species that are unable to appropriately time their reproduction in an ever-changing habitat and favor species that are able to track habitat changes. We developed a mathematical model to compare the effects of pulsed dispersal, initiated by shifts in habitat quality, with temporally continuous dispersal. We tested the effects of habitat (and population) turnover rates on metapopulation establishment, persistence, and long-term patch occupancy. Pulsed dispersal reduced patch occupancy and metapopulation longevity when habitat patches are relatively permanent. In such cases, demographic extinction was the primary form of local extinction. Conversely, when habitat patches are short-lived and new ones are frequently formed, pulsed dispersal promoted rapid colonization, increased occupancy, and prolonged metapopulation persistence. Our results show that species responsiveness to habitat disturbance is critical to metapopulation persistence, having profound implications for the species likely to persist in landscapes with altered disturbance regimes.
\end{abstract}

Keywords: conservation, dispersal behavior, disturbance regime, environmental management, habitat dynamics, metapopulation persistence.

\section{Introduction}

A challenge in metapopulation ecology and conservation management is to understand species persistence in anthropogenically and naturally disturbed landscapes. Early studies focusing on species occurrence at a landscape scale were underpinned by metapopulation ideas describing the dynamics of patch occupancy as a result of colonization

\footnotetext{
* Corresponding author; e-mail: ca.reigada@gmail.com.
}

Am. Nat. 2015. Vol. 185, pp. 183-195. (C) 2015 by The University of Chicago. 0003-0147/2015/18502-55374\$15.00. All rights reserved.

DOI: $10.1086 / 679502$ and extinction of permanent habitat patches in a fixed landscape (Levins 1969; Hanski 1999). However, recent studies have modified the assumption of a static landscape (in terms of quality and suitability of patches), inserting more realism into metapopulation studies-for example, assuming that species live in a dynamic landscape in which the habitat patches and the surrounding matrix are exposed to environmental stochasticity, habitat loss, succession or climate change (Stelter et al. 1997; Keymer et al. 2000; Amarasekare and Possingham 2001; Driscoll et al. 2013). A fully dynamic landscape could include changes in the availability, quality, and position of both usable habitat (of various types) and nonhabitat areas ("matrix") that influence dispersal. However, consistent with earlier metapopulation studies, we consider models in which the habitat areas are dynamic but the nonhabitat areas are fixed in their effect on dispersal (for a discussion of the effects of a dynamic matrix, see Blaum et al. 2012).

In a dynamic landscape, the period for which patches remain unsuitable for species colonization and the frequency at which new suitable patches appear have strong effects on persistence of populations. High sensitivity to habitat disturbance due to life history, such as altering propagule production rate and extinction proneness, makes the ability to follow landscape changes through time more dependent on species' dispersal ability (Thomas 1994; Keymer et al. 2000; DeWood et al. 2005). For instance, fast habitat turnover can reduce the rates at which individuals are spread across the landscape if individuals are not able to propagate in ephemeral habitats. Conversely, fast habitat turnover can benefit populations in which propagule production and release occur faster than habitat changes. Consequently, changes in disturbance regimes can alter community structure.

The study of the temporal structure of landscapes and disturbance regimes has important applications for habitat management. Restoration or the improvement of the quality of habitat patches and surrounding matrix can fre- 
quently alter disturbance and successional rates in landscapes and may have both direct and indirect effects on species' dispersal rates, persistence, and distribution (Blaum et al. 2012; Driscoll et al. 2013). For example, fire suppression and flood control are commonly practiced, but it is generally unknown whether and how the management of habitat dynamics may affect dispersal and subsequent metapopulation dynamics of organisms that are intrinsically linked to disturbances (e.g., through temporally pulsed dispersal).

Different dispersal strategies exist in relation to habitat condition and dynamics. Dispersal can be triggered by environmental disturbances or may even be intrinsically linked to habitat patch destruction, resulting in "pulsed dispersal." Thereby, disturbance or destruction of the habitat is not necessarily detrimental for the organisms, since they time the production of their dispersal stage with the onset of the disturbance (Bates et al. 2006; Altermatt and Ebert 2008), and emigration is triggered by the occurrence of the disturbance (Altermatt and Ebert 2010). Typical examples of organisms with pulsed dispersal are aquatic invertebrates, such as cladocerans, copepods, rotifers, and ostracods, which survive desiccation of ponds in a drought-tolerant resting and dispersal stage (Altermatt and Ebert 2008).

Dispersal may also occur immediately prior to habitat patch destruction. For instance, dispersal in many organisms is strongly influenced by behavior (Bilton et al. 2001; Crone et al. 2001; Fellous et al. 2012; Kubisch et al. 2013). In such situations, sensing changes in the environment by a few individuals can reinforce behavioral changes in others (Crone et al. 2001). Changes in dispersal behavior and dispersal-related morphology are known from organisms in which dispersal is triggered by population density, availability of resources, or isolation of the habitat and often exhibit trade-offs with other life-history traits (Hanski et al. 2006; Ahlroth et al. 2009; De Bie et al. 2012).

For many organisms, the propensity to disperse or to produce dispersal stages is correlated with the type of habitat in which they live (Southwood 1962). Although some organisms can exhibit both forms of dispersal, the numerically dominant form of dispersal within each generation (fractions of pulsed vs. continuous dispersal) will have a strong influence on the species persistence at dynamic landscapes. Species that are associated with temporary or rapidly changing habitats have generally higher levels (i.e., high fractions) of pulsed dispersal than species associated with permanent habitats (Southwood 1962). Empirical examples of such systems are agricultural landscapes in which habitable areas are frequently changed by mowing and harvesting, lands in which flood and inundation events are frequent, and early-successional communities in disturbed sites, where the habitat quality de- clines due to resource depletion and the timing for habitat to become suitable for recolonization depends on disturbance (Stelter et al. 1997; Amarasekare and Possingham 2001; Blaum et al. 2012). In all of these systems dispersal is closely linked to the state of the habitat patch, and longterm survival in such habitat systems depends on the timing of dispersal. Since long-term survival depends on an adequate dispersal strategy, strong selection on dispersal timing may be expected.

Species may be classified according to their dispersal behaviors and the longevity of habitat patches (table 1), which can affect the longevity of populations. Populations may be either short-lived (one or a few generations) or long-lived, surviving for numerous generations. If habitat turnover is low and patches are long-lived, we would expect demographic extinction to be the most frequent cause of extinction, whereas habitat patch turnover may create extinction itself as the rate of patch destruction increases. Although many of the examples studied in table 1 have focused on metapopulation occupancy and viability in a dynamic landscape, no link between different dispersal forms and the frequency with which disturbance occurs in the landscape has been investigated. We therefore asked a series of interrelated questions about how metapopulation persistence and dynamics are affected by habitat patch dynamics and the timing of dispersal events relative to habitat patch destruction. First, how do different dispersal behaviors affect the probability of population establishment at different degrees of habitat patch turnover? Second, once established, how do species' dispersal behaviors influence patch occupancy through time in a dynamic landscape? Finally, how do changes in populationhabitat turnover rates affect metapopulation persistence? We used a continuous-time, stochastic patch-occupancy model with habitat dynamics to address these questions. We draw general conclusions about how habitat management and modification of disturbance regimes facilitates or impedes metapopulation persistence.

\section{Model and Methods}

\section{The Stochastic and Mean-Field Models}

We analyzed metapopulation dynamics in landscapes composed of a finite number of ephemeral patches $(N)$ that are either suitable or unsuitable for colonization by a given species. Consequently, patches in a landscape are in one of three possible states, $S \in\{0,1,2\}$ : unsuitable and unoccupied $(S=0)$, suitable and unoccupied $(S=1)$, and suitable and occupied $(S=2)$. The dynamics of habitat suitability are determined by three parameters, $\lambda, \beta_{1}$, and $\beta_{2}$ (fig. 1). Unsuitable patches become suitable at a rate $\lambda$. Suitable patches may become unsuitable due to a mix- 


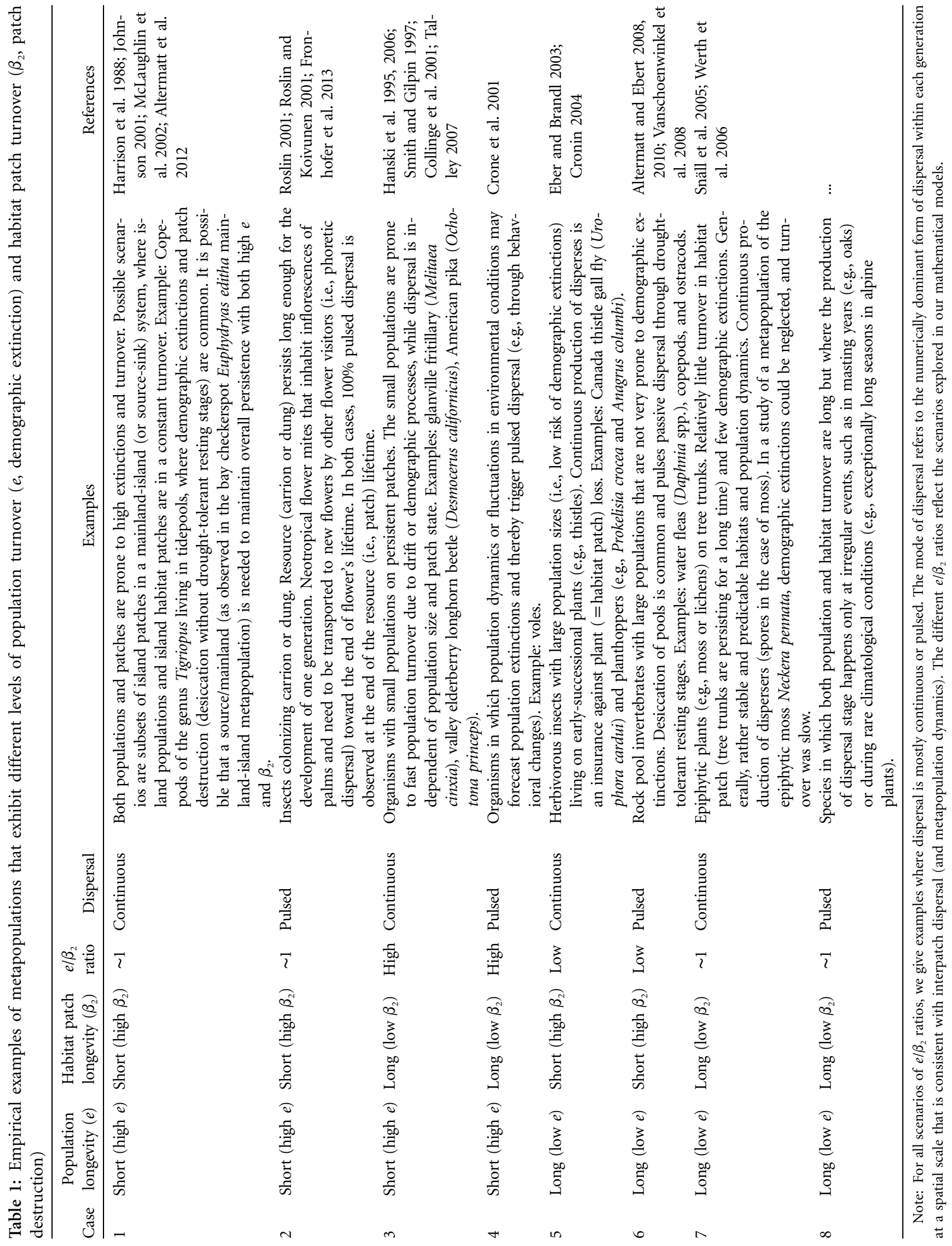

This content downloaded from 152.088.140.160 on August 11, 2017 01:05:20 AM 


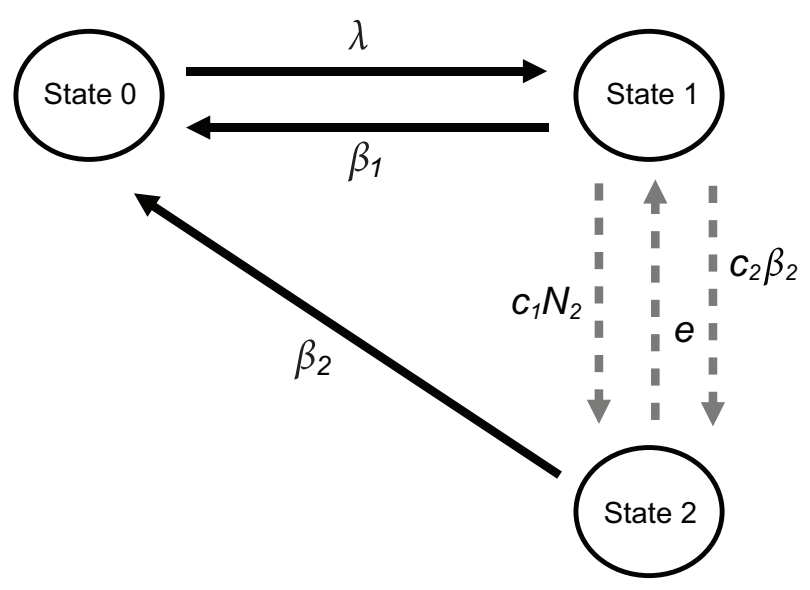

Figure 1: Transitions between patch states for a metapopulation living in a dynamic landscape. States 0,1 , and 2 correspond to patches being unsuitable and unoccupied, suitable and unoccupied, and suitable and occupied, respectively. Patch dynamics (solid black arrows) are governed by restoration rates of patches, $\lambda$, and destruction rates of patches, $\beta_{1}$ and $\beta_{2}$. Metapopulation dynamics (dashed gray arrows) are governed by colonization and extinction rates $c_{1}+c_{2} \beta_{2}$ and $e$.

ture of external and internal drivers. Hence, unoccupied suitable patches and occupied suitable patches may become unsuitable at different rates ( $\beta_{1}$ and $\beta_{2}$, respectively). When $\beta_{1}=\beta_{2}$, the dynamics of habitat suitability are independent of patch occupancy. If $\beta_{2}>\beta_{1}$, then occupancy decreases the life span of a suitable patch.

Populations residing in a suitable patch produce propagules continuously at rate $c_{1}$ and a pulse of $c_{2}$ propagules when their patch becomes unsuitable (fig. 1). The net colonization rate is

$$
c=c_{1}+c_{2} \beta_{2} .
$$

Populations in an occupied patch become extinct either because their patch becomes unsuitable or at a rate $e$ due to other sources of local extinction, including disturbances unrelated to the habitat dynamics and demographic extinction.

The stochastic model is given by a continuous-time Markov chain process whose state is characterized by the total number of unsuitable patches $\left(N_{0}\right)$, the total number of suitable but unoccupied patches $\left(N_{1}\right)$, and the total number of occupied patches $\left(N_{2}\right)$. Since all patches must be in one of these three states, $N_{0}+N_{1}+N_{2}=N$. The model exhibits two types of changes in patch state. First, there are changes in state due to empty patches becoming suitable, empty patches becoming unsuitable, occupied patches becoming unsuitable, local demographic extinction, or colonization from an occupied patch, which occur at rates $\lambda$, $\beta_{1}, \beta_{2}, e$, and $c_{1} N_{2} / N$, respectively (fig. 1 ). We interpret these rates roughly as follows: over a short time interval of length
$\Delta t$, the probability at which an unsuitable patch becomes suitable is approximately $\lambda \Delta t$; the probabilities that occupied or unoccupied patches become unsuitable are approximately $\beta_{1} \Delta t$ and $\beta_{2} \Delta t$, respectively; and the probability that an occupied suitable patch leads to colonization of an empty suitable patch is approximately $\left(c_{1} N_{1} / N\right) \Delta t$. The second type of changes in patch state occurs when an occupied patch becomes unsuitable. Pulsed dispersal results in individuals leaving the patch simultaneously and subsequently colonizing empty patches. During a pulsed dispersal event, each suitable and unoccupied patch is colonized with probability $c_{2} / N$. When $N$ is large, pulsed dispersal events lead to approximately a Poisson-distributed number of colonization events with mean $p_{1} c_{2}$.

When the number of patches $N$ is sufficiently large, the dynamics of the stochastic model are well approximated by a mean-field model (Kurtz 1978). For this mean-field model, let $p_{i}=N_{i} / N$ denote the fraction of patches in state $i=0,1,2$. The dynamics of the mean-field model are given by the following system of differential equations:

$$
\begin{aligned}
& \frac{\mathrm{d} p_{0}}{\mathrm{~d} t}=\beta_{1} p_{0}+\beta_{2} p_{2}-\lambda p_{0}, \\
& \frac{\mathrm{d} p_{1}}{\mathrm{~d} t}=\lambda p_{0}-c p_{1} p_{2}-\beta_{1} p_{1}+e p_{2}, \\
& \frac{\mathrm{d} p_{2}}{\mathrm{~d} t}=c p_{1} p_{2}-\left(e+\beta_{2}\right) p_{2} .
\end{aligned}
$$

\section{Numerical and Analytic Approaches}

We studied the mean-field and stochastic models using a mixture of analytic and numerical approaches. For the mean-field model, we examined metapopulation persistence and equilibrium occupancies using standard techniques from dynamical systems. This analysis is presented in appendix A (apps. A-C are available online). Numerical solutions for the mean-field model were computed with the DeSolve package of $\mathrm{R}$ ( $\mathrm{R}$ Development Core Team 2012).

To estimate the probability of establishment of a metapopulation for the stochastic model, we used a branching process approximation of the Markov chain when the number of patches is sufficiently large. The approximation assumes that there are sufficiently many patches that the fluctuations in $p_{1}$ around the unoccupied landscape equilibrium $p_{1}^{*}=\lambda /\left(\lambda+\beta_{1}\right)$ are sufficiently small that they can be ignored. This assumption is supported by numerical simulations of the full stochastic model. We show in appendix $B$ that the establishment probability $1-s$ can be approximated by the smallest positive fixed point of the 
following probability-generating function for the branching process:

$$
\begin{aligned}
g(s)= & \frac{1}{1+\left[c_{1} /\left(e+\beta_{2}\right)\right] p_{1}^{*}(1-s)} \\
& \times\left(\frac{e}{e+\beta_{2}}+\frac{\beta_{2}}{e+\beta_{2}} \exp \left(c_{2} p_{1}^{*}(s-1)\right)\right) .
\end{aligned}
$$

To solve for this fixed point numerically, we used the standard method of computing $g^{t}(0)$ for sufficiently large $t$ (Harris 2002).

Unlike the mean-field models, pulsed and continuous dispersal events differently impact the stochastic dynamics for a fixed colonization rate $c$. Consequently, for our analysis of the stochastic models, we introduce a parameter, $\alpha$, corresponding to the fraction of colonization events, on average, due to pulsed dispersal. The extreme of $\alpha=0$ represents species that only disperse continually during the habitat life span, and when a patch is destroyed, the population becomes extinct. Alternatively, $\alpha=1$ represents species that disperse only when disturbances occur. While some species exhibit a dominant form of dispersal (i.e., $\alpha=0$ or 1 ), some species can display both modes of dispersal. With this notation, the quantities $c_{2} \beta_{2}=$ $\alpha c$ and $c_{1}=(1-\alpha) c$ describe the relative contributions of pulsed and continuous dispersal events.

The effects of dispersal behavior and landscape dynamics on stochastic fluctuations and metapopulation viability are analyzed using numerical simulations with Gillespie's algorithm (Gillespie 1977) in R. We examined three habitat-population turnover rates in which population life span $(1 / e)$ is longer, similar, and shorter than the habitat life span $\left(1 / \beta_{2}\right)$. The measures for habitat-population turnover rates were based on empirical examples found in nature (table 1). For each parameter combination, we ran 100 simulations for 5,000 time units. For larger landscapes ( $N=1,600$ patches), the metapopulations always persisted for the entire duration of the simulation, and we analyzed the temporal changes in the number of suitable empty and suitable occupied patches using cross-correlation analyses. We concluded our analyses by examining the persistence time of smaller landscapes $(50<N<$ 1,500 patches) to identify the minimal landscape size (i.e., minimal number of patches) for metapopulation viability.

\section{Results \\ Long-Term Metapopulation Persistence and Patch Occupancy}

Long-term metapopulation persistence for the mean-field model is determined by the reproductive number $R_{0}$ of an occupied patch in a largely empty landscape. This re- productive number corresponds to the number of patches colonized by an occupied patch during its "life span" in a mostly unoccupied landscape. When this reproductive number is greater than 1 , a population in an occupied patch colonizes more than one patch before becoming locally extinct. Hence, the number of occupied patches tends to increase provided $R_{0}>1$. Conversely, when $R_{0}<1$, occupied patches do not replace themselves on average, and the metapopulation tends to move deterministically toward extinction. Landscape and population characteristics simultaneously determine the reproductive number $R_{0}$. Specifically, colonization and extinction rates regulate the propagule production by local populations and the life span of occupied patches. Habitat dynamics determine the availability of suitable habitat and trigger dispersal events. Taking these factors into account, $R_{0}$ is the product of three terms: the mean life span of an occupied habitat patch, $\tau$; the equilibrium fraction of suitable patches when the landscape is unoccupied, $s$; and the species colonization rate, $c_{1}+c_{2} \beta_{2}$.

The mean life span of an occupied habitat patch, $\tau$, represents the time before it becomes unoccupied due to either demographic extinction or a shift in habitat suitability:

$$
\tau=\frac{1}{e+\beta_{2}} .
$$

The second component, $s$, corresponds to the equilibrium fraction of suitable habitat when the landscape is unoccupied:

$$
s=\frac{\lambda}{\lambda+\beta_{1}} .
$$

Intuitively, when disturbance rates are faster than the creation of new habitat patch rates (i.e., $\beta_{1}>\lambda$ ), the amount of suitable habitat, $s$, is small and restricts colonization success. The mean number of propagules produced by an occupied patch is given by $\tau\left(c_{1}+c_{2} \beta_{2}\right)$. The reproductive number of the metapopulation is the product of $\tau, s$, and $c_{1}+c_{2} \beta_{2}$ :

$$
R_{0}=s \tau c=\frac{\lambda}{\lambda+\beta_{1}} \frac{c_{1}+c_{2} \beta_{2}}{\beta_{2}+e} .
$$

When $R_{0}>1$, the metapopulation persists at a globally stable, positive equilibrium (see app. A) given by

$$
\begin{aligned}
& \dot{p}_{0}^{*}=1-\ddot{p}_{1}^{*}-\dot{p}_{2}^{*}, \\
& \dot{p}_{1}^{*}=\frac{\beta_{2}+e}{c_{1}+c_{2} \beta_{2}}, \\
& p_{2}^{*}=1-\frac{\beta_{2}}{\lambda+\beta_{2}}-\frac{\left(\lambda+\beta_{1}\right)\left(e+\beta_{2}\right)}{\left(c_{1}+c_{2} \beta_{2}\right)\left(\lambda+\beta_{2}\right)} .
\end{aligned}
$$



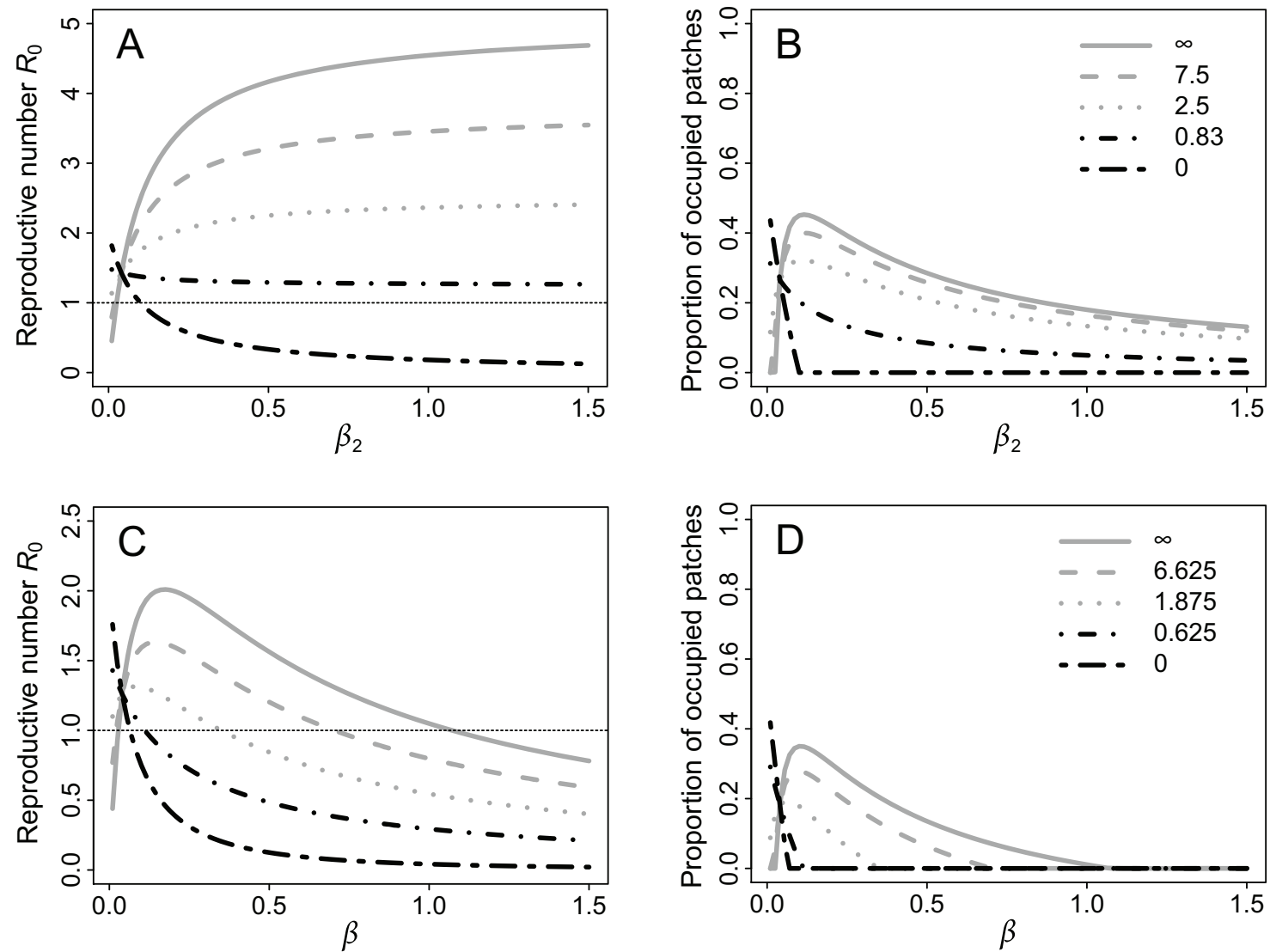

Figure 2: Metapopulation persistence and patch occupancy when only patch occupancy causes shifts in patch suitability $(A, B)$ and when shifts in habitat quality are independent of occupancy $(C, D)$. In $A$ and $B$, the reproductive number for an individual patch during its life span and the equilibrium patch occupancy are plotted as functions of the rate $\beta_{2}$ at which occupied patches become unsuitable. Gray lines represent cases where $c_{2}>c_{1} / e$, and black lines represent cases where pulsed dispersal produces fewer colonizers than continuous dispersal $\left(c_{2}<c_{1} / e\right)$. In $C$ and $D$, reproductive number for an individual patch during its life span and patch occupancy are plotted as functions of the rate at which suitable patches become unsuitable $\left(\beta_{1}=\beta_{2}=\beta\right)$. Gray lines represent cases where $c_{2}>c_{1} / e+c_{1} / \lambda$, and black lines represent cases where $c_{2}<c_{1} / e+c_{1} / \lambda$. The horizontal dotted gray lines in $A$ and $C$ are the thresholds for metapopulation persistence. Parameter values: $\lambda=0.3 ; e=0.1 ; c_{1}=0,0.5,1,1.5 ;$ and $c_{2}=5,3.75,2.5,1.25,0$.

When $R_{0}<1$, the metapopulation reaches extinction for all initial conditions (see app. A). Equations (5) and (6) imply that the reproductive number and the equilibrium patch occupancy $\left(p_{2}^{*}\right)$ increase with colonization rates $c_{1}$ and $c_{2}$, decrease with local extinction rate $e$, and increase with the rate $\lambda$ at which unsuitable habitat becomes suitable.

Changes in patch states may occur when patch suitability depends on the availability of a depleted resource (i.e., $\beta_{1}=0$ but $\beta_{2}>0$ ). In this kind of dynamic landscape, $1 / \beta_{2}$ is the mean time the population draws down the resource, and $1 / \lambda$ is the mean recovery time for the resource. For this dynamic landscape, the effect of $\beta_{2}$ on metapopulation persistence and equilibrium occupancy depends on the mean number of colonizers $\left(c_{1} / e\right)$ produced by a patch prior to demographic extinction and the mean number of colonizers $\left(c_{2}\right)$ produced by a pulsed dispersal event. When pulsed dispersal produces more colonizers than continuous dispersal (i.e., $c_{2}>c_{1} / e$ ), increasing the rate at which habitat becomes unsuitable increases the metapopulation's reproductive number (fig. $2 A$ ). Intuitively, the loss of colonizers prior to the pulsed dispersal event is overcompensated for by the increased frequency of pulsed dispersal events. Consistent with the effect of $\beta_{2}$ on $R_{0}$, increasing $\beta_{2}$ increases the equilibrium patch occupancy at low values of $\beta_{2}$ (fig. $2 B$ ). However, at high levels of $\beta_{2}$, increasing $\beta_{2}$ reduces the persistence time of occupied patches enough to cause a reduction in equilibrium patch occupancy. On the other hand, when continuous dispersal produces more colonizers than pulsed dispersal $\left(c_{1} / e>c_{2}\right)$, increasing $\beta_{2}$ decreases both $R_{0}$ and the equilibrium patch frequency. 


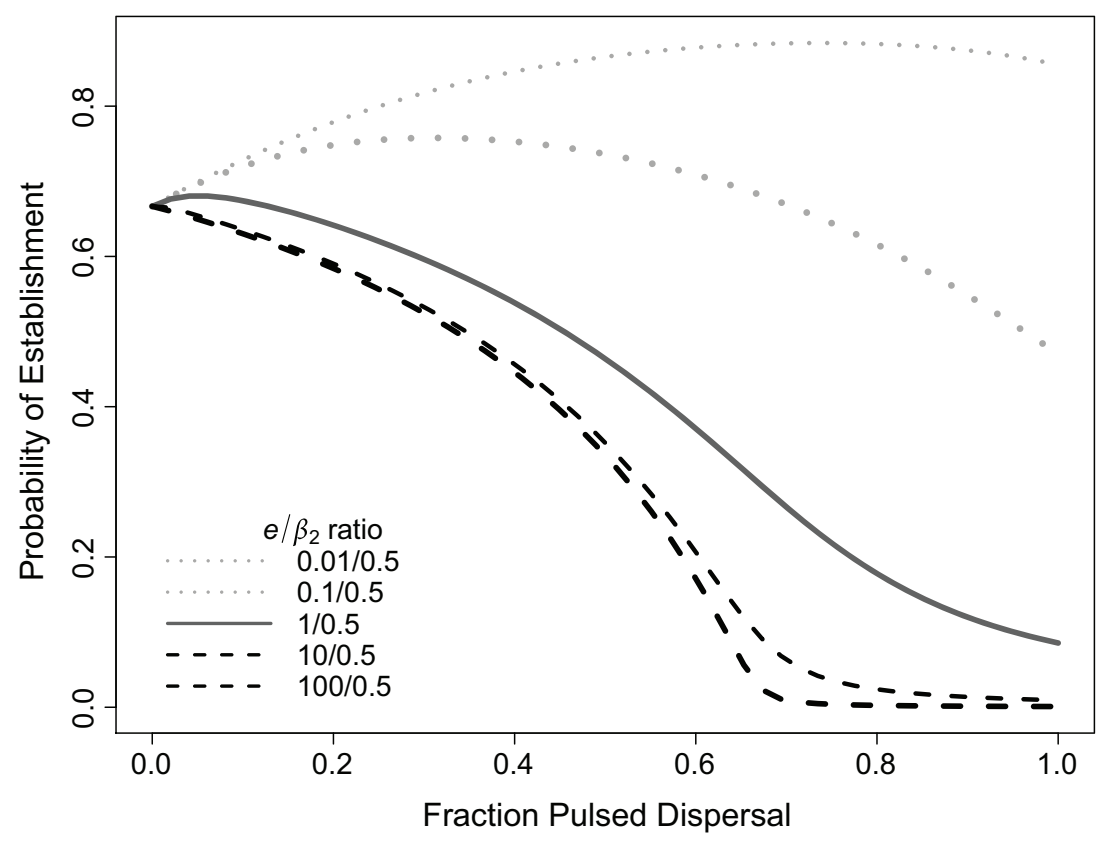

Figure 3: Establishment probability as a function of the fraction of colonization due to pulsed dispersal. Different lines correspond to different ratios of habitat life span to population life span $\left(e / \beta_{2}\right)$ : for light gray dotted lines habitat life span is shorter than population life span, and for black dashed lines population life span is shorter than habitat life span. Parameter values: $e=0.001,0.1,1,10,100 ; \beta_{2}=$ $0.5 ; \lambda=0.3 ; R_{0}=3$; and $c_{2}=\alpha\left(c_{1} / \beta_{2}\right)$, where $\alpha$ is the fraction of colonization events due to pulsed dispersal.

An alternate habitat dynamic occurs when changes in habitat suitability are driven purely by exogenous factors and all suitable patches, unoccupied or occupied, experience the same habitat shift rate. For this dynamic landscape, the effect of $\beta=\beta_{1}=\beta_{2}$ on the metapopulation reproductive number and equilibrium patch occupancy depends in a subtle manner on the mean number of colonizers produced during a dispersal event $\left(c_{2}\right)$, the mean number of colonizers produced prior to a demographic extinction event $\left(c_{1} / e\right)$, and the mean number of colonizers lost during the time a patch remains unsuitable $\left(c_{1} / \lambda\right)$. When $c_{2}>c_{1} / e+c_{1} / \lambda, R_{0}$ and $p_{2}^{*}$ exhibit a humped-shaped relationship with $\beta$ (fig. $2 C, 2 D$ ), increasing at low $\beta$ and decreasing at high $\beta$. Under these circumstances, the metapopulation persists only at intermediate rates of suitable patches becoming unsuitable. On the other hand, when $c_{2}<c_{1} / \lambda+c_{1} / e$, increasing rates at which patches become unsuitable always decreases $R_{0}$ and $p_{2}^{*}$, and there is a critical value above which metapopulation persistence is no longer possible.

\section{Establishment, Stochastic Fluctuations, and Persistence in Finite Landscapes}

While the mean-field model provides useful insights into persistence and long-term patch occupancy for landscapes with a large number of patches, stochastic effects play a significant role in metapopulations with fewer patches. Stochastic effects also generate fluctuations of varying magnitudes around the mean-field equilibrium and ultimately determine metapopulation viability in landscapes with a finite number of patches (see fig. C1; figs. C1, C2 are available online). Using the stochastic model, we analyzed how different dispersal behaviors influence establishment, the covariance structure of fluctuations between suitable unoccupied and occupied patches on the event of establishment (i.e., spatiotemporal variance in patch occupancy), and persistence times for established metapopulations.

Metapopulation Establishment Probability. Using the analytical approximation described in "Model and Methods," our analysis (see app. B) reveals that the effect of pulsed dispersal on metapopulation establishment depends on the relative lengths of population life span $(1 / e)$ and habitat life span $\left(1 / \beta_{2}\right)$. When the habitat life span is short relative to the population life span (cases 5 and 6 in table 1), pulsed dispersal has a positive effect on metapopulation establishment; metapopulations with an intermediate propensity for pulsed dispersal are most likely to establish (gray dotted curves in fig. 3). In contrast, when the habitat life span is long relative to the population life span (cases 3 
and 4 in table 1), metapopulations with any propensity for pulsed dispersal are less likely to establish (black dashed curves in fig. 3). These differences do not stem from differences in the mean colonization rates, as they are unaffected by the fraction of the population exhibiting pulsed dispersal. Instead, these differences stem from the variation in the number of patches colonized by an occupied patch during its life span; greater variation in the number of patches colonized results in lower establishment probabilities (Lloyd-Smith et al. 2005), as we now explain. When the habitat life span is long relative to the population life span (cases 3 and 4 in table 1), pulsed dispersal leads to greater variation in the number of patches colonized (see app. B). Intuitively, the reproductive number of a patch $R_{0}$, in this case, is achieved by most occupied patches becoming extinct prior to colonizing other patches, while a few occupied produce large, pulsed colonization events. Consequently, when habitat life span is long, pulsed dispersal creates greater variation in propagules produced and thereby decreases the likelihood of establishment. Conversely, when the habitat life span is short relative to the population life span, most occupied patches produce a somewhat similar number of pulsed dispersers. Subsequently, pulsed dispersal can produce less variation in the number of colonized patches and can increase the likelihood of establishment.

Fluctuations in Patch Occupancy. When a metapopulation has established in a landscape, it can persist for a long time and exhibit metastable behavior. During this period, patch occupancies tends to fluctuate around the equilibrium $p_{0}^{*}, p_{1}^{*}, p_{2}^{*}$ of the mean-field model. Using numerical simulations, we examined how population life spans and habitat life spans in conjunction with dispersal mode influence the spatial and temporal covariance structure of these fluctuations. More specifically, we considered three landscape scenarios (see fig. C2) in which habitat life spans are shorter (cases 5 and 6 in table 1), longer (cases 3 and 4 in table 1), and similar (cases $1,2,7$ and 8 in table 1 ) to population life spans, that is, $1 / \beta_{2} \ll 1 / e, 1 / \beta_{2} \gg 1 / e$, and $1 / \beta_{2} \approx 1 / e$, respectively. When habitat life spans are shorter than population life spans, patch destruction is the main factor reducing patch occupancy. On the other hand, when habitat life spans are longer relative to population life spans, demographic extinction of local populations is the main factor reducing patch occupancy (fig. C2).

When habitat life span is long relative to population life span, the variance in patch occupancy increases with the frequency of pulsed dispersal (black dashed line in fig. $4 A)$. As in the case of metapopulation establishment, the mean colonization rate for pulsed dispersers is achieved by rare yet pronounced pulsed dispersal events. Thereby, pulsed dispersal increases the temporal variance in patch
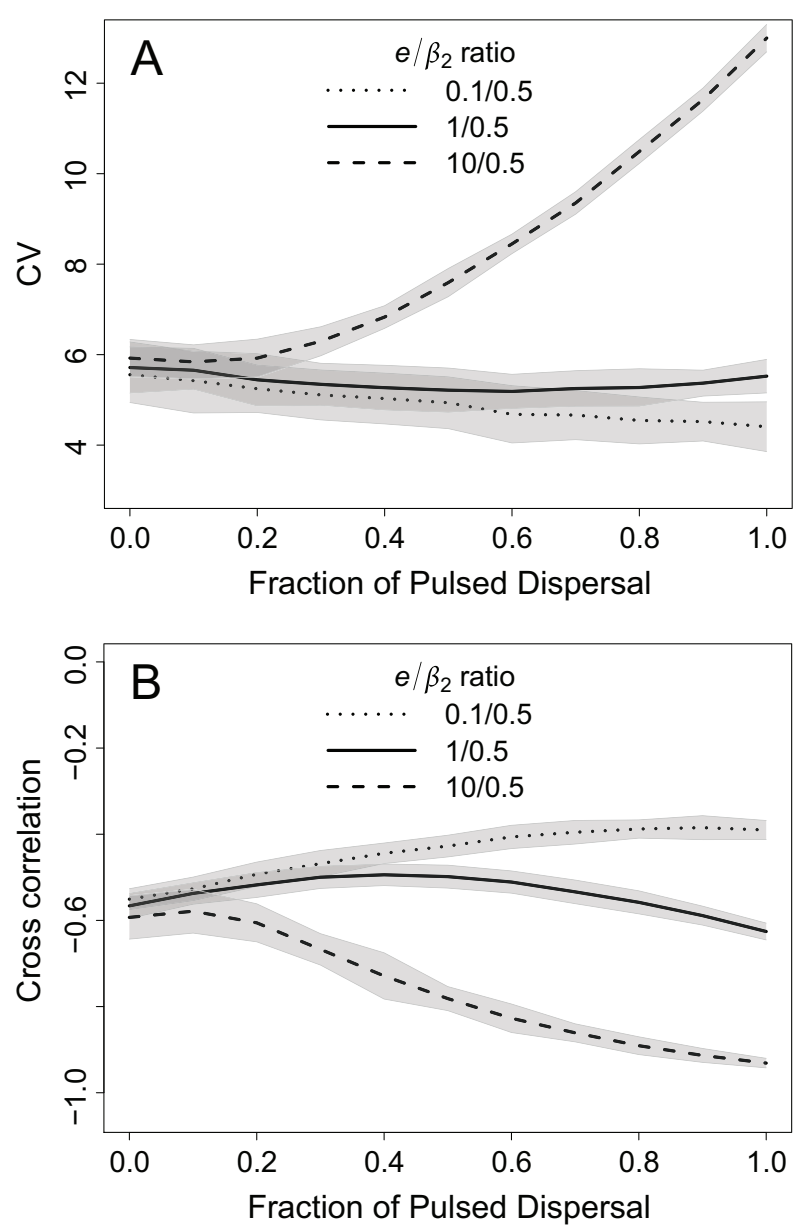

Figure 4: Covariance structure of in-patch occupancy for the stochastic model. Shown are estimated variance in patch occupancy $(A)$ and temporal synchrony between unoccupied and occupied patches (cross-correlation coefficients; $B$ ) as a function of the fraction of colonization due to pulsed dispersal. Dotted, solid, and dashed black lines represent habitat life spans shorter than population life spans, same rates of habitat life spans and population life spans, and population life spans shorter than habitat life spans, respectively. Light gray regions correspond to the interquartile ranges for 100 simulations in 1,000 time units. Parameter values: $N=1,600, e=$ $\{0.1,1,10\}, \beta_{1}=\beta_{2}=0.5, \lambda=0.3, R_{0}=3, c_{1}=R_{0}\left(e+\beta_{2}\right) / p_{1}^{*}$, and $c_{2}=c_{1} / \beta_{2}$.

occupancy, and changes in suitable occupied patches and unoccupied patches are tightly coupled and negatively correlated; reductions in occupied patches typically correspond to increases in unoccupied suitable patches (black dashed line in fig. $4 B$ ).

When average habitat life span is shorter than population life span, pulsed dispersal occurs more frequently and is balanced by higher colonization rates, which buffers the effects of patch disturbance. Under these circumstances, pulsed dispersal decreases the variation in patch 
occupancy (gray dotted line in fig. 4A). Although negatively correlated, changes in occupied and unoccupied patch states becomes less coupled since loss of a patch is followed by high production of colonizers, making changes in the number of occupied patches higher than changes in unoccupied ones (gray dotted line in fig. 4B). This decoupling reduces variation in patch occupancy.

Metapopulation Viability. To address how dispersal mode influences metapopulation viability, we considered an established metapopulation starting at patch occupancy equilibrium $p_{0}^{*}, p_{1}^{*}, \ddot{p}_{2}^{*}$ of the mean-field model and computed the mean of persistence time across 100 replicates. This was done for a range of landscape sizes. Figure $5 \mathrm{~A}$ shows how the frequency of pulsed dispersal and landscape size influence persistence in landscapes with long habitat life spans relative to population life spans (high $e / \beta_{2}$ ratio, cases 3 and 4 in table 1). Intuitively, mean persistence time increases with landscape size. When pulsed dispersal is an uncommon dispersal mode, mean persistence time increases and saturates rapidly with landscape size, and the critical landscape size is approximately 200 patches. However, when pulsed dispersal is the most common dispersal mode, mean persistence time increases gradually with landscape size, even at large patch numbers. Intuitively, pulsed dispersal in environments where populations are highly prone to extinctions results in greater fluctuations in the metapopulation dynamics and, consequently, in shorter persistence times.

To examine the effect of landscape size, habitat turnover, and dispersal mode on metapopulation viability, we defined the minimum viable metapopulation size to be the minimum number of patches resulting in a $90 \%$ chance of a metapopulation persisting at least 5,000 time steps. When habitat life span is smaller than expected population life span (cases 5 and 6 in table 1), pulsed dispersal allows high persistence at small landscape sizes (gray dotted curve in fig. $5 B$ ). Alternatively, for long habitat life spans relative to population life spans (high $e / \beta_{2}$ ratios), metapopulations displaying a high fraction of pulsed dispersal persist only in larger landscape sizes (black dashed curve in fig. $5 B)$.

\section{Discussion}

The majority of metapopulation studies predict that ephemeral or small habitat patches have a negative effect on metapopulation persistence (Lande 1987; Tilman et al. 1994; Bascompte and Solé 1996; Gyllenberg and Hanski 1997; Hill and Caswell 1999). These predictions, however, are largely based on the assumption that disturbances and dispersal/colonization dynamics are uncorrelated (Keymer et al. 2000; Amarasekare and Possingham 2001; DeWoody
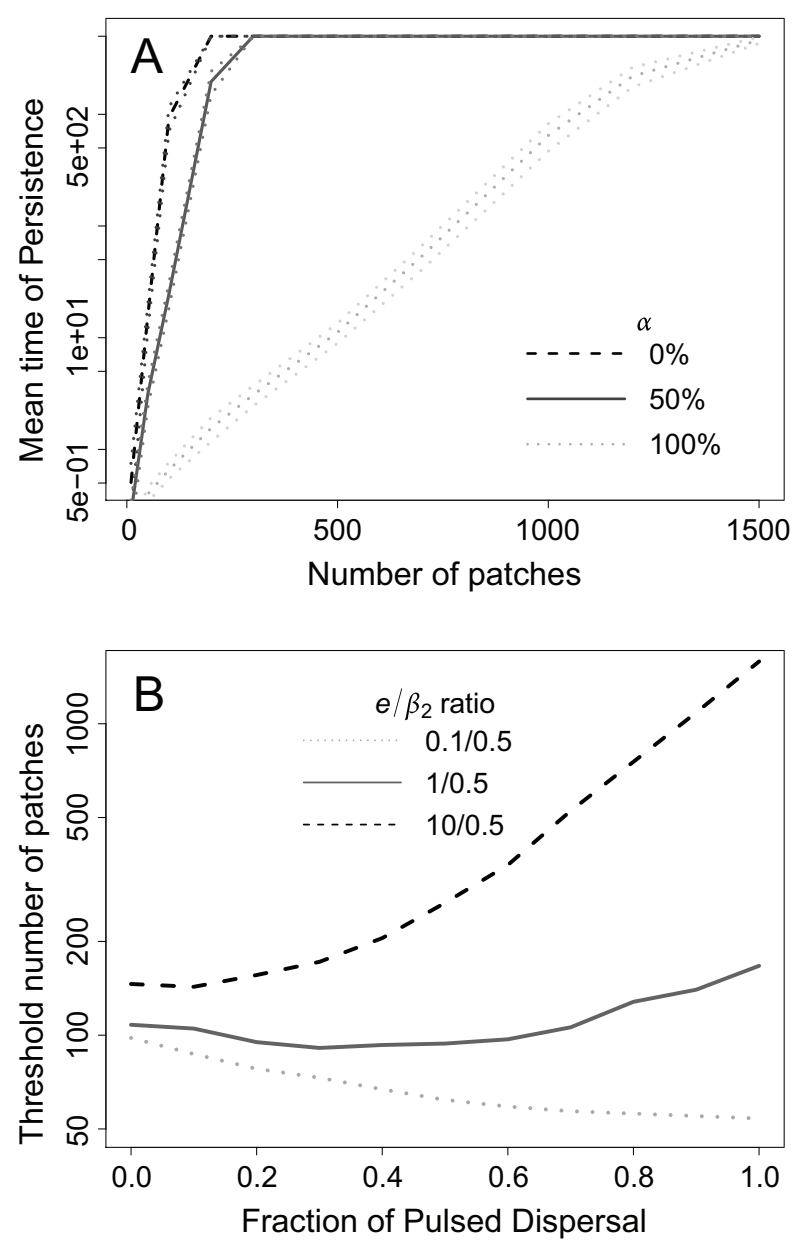

Figure 5: Influences of dispersal mode, habitat life span, and population life span on metapopulation viability and critical landscape size. A, Mean ( $95 \%$ confidence interval) of persistence time for population life spans shorter than habitat life spans $\left(e / \beta_{2}=10 / 0.5\right)$ as a function of different landscape size for $0 \%, 50 \%$, and $100 \%$ fractions of pulsed dispersal. $B$, Mean of habitat availability threshold for landscapes giving metapopulation persistence in $90 \%$ of replicates as a function of the fraction of colonization occurring through pulsed dispersal. In $B$, different curves represent variations in critical size of a dynamic landscape for different levels of $e / \beta_{2}$ ratios: the light gray dotted line corresponds to a habitat life span shorter than a population life span, the dark gray solid line corresponds to equal habitat life span and population life span, and the black dashed line corresponds to a population life span shorter than a habitat life span. Lines in $A$ and $B$ represent the result of means of 100 simulations during 5,000 time units. Parameter values: $e=10, R_{0}=3, \beta_{1}=$ $\beta_{2}=0.5, \lambda=0.3, c_{1}=R_{0}\left(e+\beta_{2}\right) / p_{1}^{*}$, and $c_{2}=\alpha\left(c_{1} / \beta_{2}\right)$, where $\alpha$ is the fraction of colonization events due to pulsed dispersal.

2005; Xu et al. 2006). Modeling habitat disturbance and species' dynamical processes separately is a straightforward way to understand extinction processes (in terms of species' life history) and patch destruction factors (Fahrig 1992; Keymer et al. 2000; Amarasekare and Possingham 
2001; DeWoody et al. 2005). However, in many systems the effects of patch disturbance and the timing of dispersal are not independent (Bowler and Benton 2005; see also table 1). Species inhabiting naturally disturbed habitats often disperse in response to changes in habitat quality in a manner that enhances their survival in these environments (Dennis et al. 2003; Bowler and Benton 2005).

Although the existence of pulsed dispersal has been documented in empirical studies (Crone et al. 2001; Bates et al. 2006; Altermatt and Ebert 2010; see also table 1), theoretical work has focused only on dispersers originating from patches before the actual occurrence of disturbances. Such dispersal has been modeled commonly in a continuous way, neglecting the "pulsed release" of dispersers when a patch is destroyed. Here, we analyzed mean-field and stochastic models to study how continuous and pulsed dispersal affects metapopulation persistence and patch occupancy at different habitat and population turnover rates. Our chosen set of model parameters-especially the suite of chosen habitat and patch turnover rates-reflect a large and realistic range of natural systems (see table 1 for examples). We also assessed the impact of population-habitat turnover rates on metapopulation viability, motivated by different examples of dispersal behavior and population life span related to the habitat in which species live.

\section{Metapopulation Establishment and Persistence in Highly Disturbed Landscapes}

A necessary condition for metapopulation establishment and long-term persistence is that the reproductive number of populations is greater than 1 . This reproductive number can be interpreted as the "infective" characteristic of a particular metapopulation to expand its range across the landscape and can be used as a criterion to define the invasion potential for populations given their colonization-extinction dynamic and the landscape's temporal structure (Keymer et al. 2000; Lloyd-Smith et al. 2005). Previous theoretical studies have used $R_{0}$ to define a threshold for particular habitat loss and restoration turnover rates in which metapopulation persistence becomes impossible (Keymer et al. 2000; DeWoody et al. 2005; Xu et al. 2006). In these models, the impact of habitat loss due to disturbances is directly related to the reduction in the number of local populations that contribute propagules to maintain colonization rates that are higher than extinction rates. However, these models have neglected the effects of pulsed dispersal on $R_{0}$.

The occurrence of pulsed dispersal helps to maintain $R_{0}$ higher than 1 when the number of propagules released after disturbance is capable of maintaining a high number of occupied patches, even at high disturbance rates. However, our results show that the long-term patch occupancy is dependent not only on $R_{0}$ but also on the recovery rates of habitats, which need to be high enough to provide a minimal number of patches for metapopulation viability. For cases in which occupancy of patches leads to occupied patches becoming unsuitable due to resource depletion, higher depletion rates favor populations with pulsed dispersal as their dominant form of dispersal. For such populations, higher depletion rates always increase reproductive numbers and increase long-term patch occupancy provided depletion rates are not too high (fig. $2 A, 2 B$ ). Conversely, when suitable occupied and suitable empty patches are equally subject to changes in suitability, the amount of propagule releases by pulsed dispersal overcompensates for the low colonization rates prior to habitat destruction (fig. 2C, 2D). However, in this case the increasing destruction of patches should be followed by high restoration rates of patches to maintain $R_{0}$ higher than 1 and a minimal metapopulation occupancy. If the rates of patch restoration are slower than the rates of habitat destruction, the metapopulation can easily become extinct. These results call for attention in the context of defining the optimal disturbance frequency at the landscape level, which varies according to the kind of patch disturbance in which landscape is exposed and can have different consequences for persistence of species, especially when they release different propagule fractions during and after patch disturbance.

The interplay between population reproductive number and dispersal behavior is essential to understand colonization rates and patch occupancy during invasion processes in landscapes composed of ephemeral patches (McArthur and Wilson 1967; Kot and Lewis 1996). Studies have shown that successful invasion is positively correlated with high dispersal frequency and a large number of individuals sent during dispersal events (Jules et al. 2002; Schreiber and Lloyd-Smith 2009). Extending these results to our dynamic landscape context, we found that when dispersal is linked to disturbance frequency, high levels of habitat turnover lead to more propagules released, increasing the probability of establishment success of invasive species in the new landscape. In this form, the increase in disturbance frequencies can promote a successful introduction and spread of invasive species across landscapes, especially when invasive species are capable of exhibiting pulsed dispersal and the frequency of patch turnover is high.

For already-established metapopulation systems, the key factor determining species persistence in dynamic landscapes is their capability to maintain per-patch colonization rates greater than disturbance rates (Keymer et al. 2000; Hastings 2003). Populations that display only temporally continuous dispersal are less tolerant to high frequency disturbance regimes, since increased disturbances negatively affect the population life span and make ex- 
tinction rates higher than colonization rates. Conversely, when the predominant form of population dispersal is pulsed, it creates a positive link between dispersal and disturbance in that colonization rates become higher when rates of habitat loss are higher, ensuring a minimum level of patch occupancy for metapopulation persistence. Therefore, ephemeral landscapes are not always detrimental for metapopulations.

\section{Short-Lived Populations Inhabiting Dynamic Landscapes}

We demonstrated the importance of positive links between dispersal and disturbance for population persistence when the life span of habitat is shorter than population life span. However, for some biological systems population life span can be shorter than habitat life span. For example, human interventions or natural environmental factors can affect successional and other processes, such that the generation of new habitats is reduced. Hence, species specialized in tracking successional habitat can be driven to extinction in managed landscapes, where the creation of new habitat is practically zero (Thomas 1994; Stelter et al. 1997). For species living in ephemeral habitats and in which dispersal is tightly linked to the disturbance of habitat (pulsed dispersal dominates), the colonization of new patches becomes difficult, and species may eventually become extinct at the metapopulation level when population turnover is faster than habitat turnover.

Our results show that when patch disturbance is the main factor promoting changes in patch occupancy, the metapopulation dynamic becomes close to a source-sink dynamic and high occupancy is achieved when colonization events are promoted by the occurrence of habitat disturbances. Conversely, at high disturbance frequencies, occupied patches act as sources of new dispersers, and high propagule release during disturbance is important to increase patch occupancy and the probability of persistence. This is also distinct from the case where the main factor causing reduction in patch occupancy is demographic extinction, in which case the dispersal and colonization rates are supported by the number of populations surviving disturbances. Then the metapopulation dynamic becomes close to classic metapopulation dynamics, and the continuous propagule release during patch occupancy balances the negative effects of population extinctions.

Shifts between patch disturbance and demographic extinction domains can happen in natural landscapes due to many external drivers already mentioned, which are then interchanging the metapopulation organization of the system. Such shifting effects on metapopulation organization have already been documented in previous studies (Stelter et al. 1997; Hastings 2003). In agreement with these studies, our findings show that changes in the timing of disturbance occurrences related to population life span deeply affected the criterion for minimum per-patch colonization rates that are sufficient to maintain a metapopulation. The negative effects coming from the shift between disturbance domain to demographic extinction domain governing patch occupancy are likely to be more drastic for populations with dispersal linked to habitat changes. For these cases, the advantages of high fractions of pulsed dispersal on population colonization rates are restricted to metapopulations living in landscapes composed of a high number of habitat patches, when pulsed dispersal ensures colonization rates higher than extinction rates (fig. 5). In small landscapes, we found high fluctuations and, consequently, low patch occupancy and high extinction risks.

\section{Implications for Conservation and Management of Species}

Landscapes composed of ephemeral patches are not only diverse and contain specialized communities but also depend on a proper adoption of management strategies that balance the positive and negative effects of patch destruction, creation, and suppression of disturbances (Stelter et al. 1997; Keymer et al. 2000; Hastings 2003). The modification of habitat patches and the surrounding areas can extensively change the landscape and make the habitat and matrix more or less hostile for species. For successful landmanagement practices, it is necessary to understand critical processes influencing species presence in dynamic landscapes. Here, we for the first time specifically addressed the role of dispersal behavior relative to disturbance and how it shapes the relationship between populations and their habitats in situations when patch and population turnover are coupled or not.

In table 1, we show how metapopulation systems can differ with respect to population/habitat longevity rates and dispersal modes. The management of systems in which demographic extinction rates are similar to (cases 1, 2, 7, and 8 in table 1) or higher than (cases 3 and 4 in table 1) habitat patches' turnover rates varies in the conservation plan required to ensure persistence, depending on species' dispersal behavior. For species exhibiting continuous dispersal during the habitat life span, efforts to improve habitat quality can promote dispersal and positive effects on metapopulation dynamics. However, if the focal species has a predominantly pulsed dispersal behavior, it is necessary to invest in increasing the amount of habitat to maintain the species at the landscape level.

For cases in which habitat turnover is faster than demographic extinction (cases 5 and 6 in table 1), frequent habitat manipulation/restoration can increase the persistence probability for species that are capable of pulsed dispersal. For example, for landscapes mosaics formed by short-term crop systems, the manipulation of spatiotem- 
poral distribution of host plants can be a useful strategy to control and reduce the incidence of pest insects that disperse in pulses after harvest. When these species are capable of using alternative host plants as refuge during the absence of the primary host plant, creating gaps in time between the succession of crops can restrict the spread and consequently the economic damage caused by these species (Fitt et al. 1995; Tilman et al. 2009). The different practices for management of burnt wood after fire occurrence is another example of how habitat disturbs and dispersal can affect occupancy of species. The management of burned forest areas also affects the recovery rate by affecting the number of seed predators that destroy the dispersal propagules. Studies have shown that postfire polices of removal of burnt trunks and remaining debris from burnt areas can increase the vegetation restoration rates by reducing the predation of seed from these areas (PuertaPiñero et al. 2010).

Our theoretical study calls attention to the importance of shift between organizational systems of metapopulation dynamics for species with different dispersal behavior. Since in real landscapes the time and duration of disturbances can be quite variable, populations can face temporal changes between dynamical regimes or live in spatialtemporal mosaics, with mixtures of the two dynamical regimes. Different dispersal behavior may or may not facilitate species persistence, depending on landscape features such as size and disturbance regime. Our general results can be used for a wide range of species (table 1) and help to estimate how anthropogenic and natural changes of the temporal structure of landscapes can influence metapopulation viability.

\section{Acknowledgments}

Financial support for this project was provided by Fundação de Amparo á Pesquisa do Estado de São Paulo (FAPESP; postdoctoral fellowship; 2012/01189-7). C.R. was supported by FAPESP and Coordenação de Aperfeiçoamento de Pessoal de Nível Superior (CAPES)/Programa Nacional de Pós Doutorado (PNPD). F.A. was supported by the Swiss National Science Foundation (31003A_135622 and PP00P3_150698). M.H. was supported by the US National Science Foundation (NSF; DEB-0639885) and his Agricultural Experiment Station project. S.J.S. was supported by the NSF (grants EF-0928987 and DMS-1022639).

\section{Literature Cited}

Ahlroth, P., R. Alatalo, and J. Suhonen. 2009. Reduced dispersal propensity in the wingless waterstrider Aquarius najas in a highly fragmented landscape. Oecologia (Berlin) 162:323-330.
Altermatt, F., and D. Ebert. 2008. The influence of pool volume and summer desiccation on the production of the resting and dispersal stage in a Daphnia metapopulation. Oecologia (Berlin) 157:441452.

2010. Populations in small, ephemeral habitat patches may drive dynamics in a Daphnia magna metapopulation. Ecology 91: 2975-2982.

Altermatt, F., A. Bieger, and S. Morgan. 2012. Habitat characteristics and metapopulation dynamics of the copepod Tigriopus californicus. Marine Ecology Progress Series 468:85-93.

Amarasekare, P., and H. Possingham. 2001. Patch dynamics and metapopulation theory: the case of successional species. Journal of Theoretical Biology 209:333-344.

Bascompte, J., and R. Solé. 1996. Models of habitat fragmentation. Pages 127-150 in J. Bascompte and R. Solé, eds. Modeling spatiotemporal dynamics in ecology. Springer, Berlin.

Bates, A. J., J. P. Sadler, and A. P. Fowles. 2006. Condition-dependent dispersal of a patchily distributed riparian ground beetle in response to disturbance. Oecologia (Berlin) 150:50-60.

Bilton, D. T., J. R. Freeland, and B. Okamura. 2001. Dispersal in freshwater invertebrates. Annual Review of Ecology and Systematics 32:159-181.

Blaum, N., M. Schwager, M. C. Wichmann, and E. Rossmanith. 2012. Climate induced changes in matrix suitability explain gene flow in a fragmented landscape - the effect of interannual rainfall variability. Ecography 35:650-660.

Bowler, D. E., and T. G. Benton. 2005. Causes and consequences of animal dispersal strategies: relating individual behavior to spatial dynamics. Biological Reviews 80:205-225.

Collinge, S. K., M. Holyoak, C. B. Barr, and J. T. Marty. 2001. Riparian habitat fragmentation and population persistence of the threatened valley elderberry longhorn beetle in central California. Biological Conservation 100:103-113.

Crone, E. E., D. Doak, and J. Pokki. 2001. Ecological influences on the dynamics of a field vole metapopulation. Ecology 82:831-843.

Cronin, J. 2004. Host-parasitoid extinction and colonization in a fragmented prairie landscape. Oecologia (Berlin) 139:503-514.

De Bie, T., L. De Meester, L. Brendonck, K. Martens, B. Goddeeris, D. Ercken, H. Hampel, et al. 2012. Body size and dispersal mode as key traits determining metacommunity structure of aquatic organisms. Ecology Letters 15:740-747.

Dennis, R. L. H., T. G. Shreeve, and H. Van Dyck. 2003. Towards a functional resource-based concept for habitat: a butterfly biology viewpoint. Oikos 102:417-426.

DeWoody, Y. D., Z. Feng, and R. K. Swihart. 2005. Merging spatial and temporal structure within a metapopulation model. American Naturalist 166:42-55.

Driscoll, D. A., S. C. Banks., P. S. Barton., D. B. Lindenmayer, and A. L. Smith. 2013. Conceptual domain of the matrix in fragmented landscapes. Trends in Ecology and Evolution 28:605-613.

Eber, S., and R. Brandl. 2003. Regional patch dynamics of Cirsium arvense and possible implications for plant-animal interactions. Journal of Vegetation Science 14:259-266.

Fahrig, L. 1992. Relative importance of spatial and temporal scales in a patchy environment. Journal of Theoretical Population Biology 41:300-314.

Fellous, S., A. Duncan, A. L. Coulon, and O. Kaltz. 2012. Quorum sensing and density-dependent dispersal in an aquatic model system. PLoS ONE 7:e48436.

Fitt, G. P., M. L. Dillon, and J. G. Hamilton. 1995. Spatial dynamics 
of Helicoverpa populations in Australia: simulation modeling and empirical studies of adult movement. Computers and Electronics in Agriculture 13:177-192.

Fronhofer, E. A., E. B. Sperr, A. Kreis, M. Ayasse, H. J. Poethke, and M. Tschapka. 2013. Picky hitch-hikers: vector choice leads to directed dispersal and fat-tailed kernels in a passively dispersing mite. Oikos 122:1254-1264.

Gillespie, D. T. 1977. Exact stochastic simulation of coupled chemical reactions. Journal of Physical Chemistry 81:2340-2361.

Gyllenberg, M., and I. Hanski. 1997. Habitat deterioration, habitat destruction, and metapopulation persistence in a heterogenous landscape. Theoretical Population Biology 52:198-215.

Hanski, I. 1999. Metapopulation ecology. Oxford University Press, Oxford.

Hanski, I., J. Pöyry, T. Pakkala, and M. Kuussaari. 1995. Multiple equilibria in metapopulation dynamics. Nature 377:618-621.

Hanski, I., M. Saastamoinen, and O. Ovaskainen. 2006. Dispersalrelated life-history trade-offs in a butterfly metapopulation. Journal of Animal Ecology 75:91-100.

Harris, T. 2002. The theory of branching processes. Courier Dover, Mineola, NY.

Harrison, S., D. D. Murphy, and P. R. Ehrlich. 1988. Distribution of the Bay checkerspot butterfly, Euphydryas editha bayensis: evidence for a metapopulation model. American Naturalist 132:360-382.

Hastings, A. 2003. Metapopulation persistence with age-dependent disturbance or succession. Science 310:1525-1526.

Hill, M. F., and H. Caswell. 1999. Habitat fragmentation and extinction thresholds on fractal landscapes. Ecology Letters 2:121127.

Johnson, M. P. 2001. Metapopulation dynamics of Tigriopus brevicornis (Harpacticoida) in intertidal rock pools. Marine Ecology Progress Series 211:215-224.

Jules, E. S., M. J. Kauffman, W. D. Ritts, and A. L. Carroll. 2002. Spread of an invasive pathogen over a variable landscape: a nonnative root rot on Port Orford cedar. Ecology 83:3167-3181.

Keymer, J. E., P. A. Marquet, J. X. Velasco-Hernández, and S. A. Levin. 2000. Extinction thresholds and metapopulation persistence in dynamic landscapes. American Naturalist 156:478-494.

Kot, M., and M. A. Lewis. 1996. Dispersal data and the spread of invading organisms. Ecology 77:2027-2042.

Kubisch, A., R. D. Holt, H. J. Poethke, and E. A. Fronhofer. 2013. Where am I and why? synthesizing range biology and the ecoevolutionary dynamics of dispersal. Oikos 123:5-22.

Kurtz, T. G. 1978. Strong approximation theorems for density dependent Markov chains. Stochastic Processes and Their Applications 6:223-240.

Lande, R. 1987. Extinction thresholds in demographic models of territorial populations. American Naturalist 130:624-635.

Levins, R. 1969. Some demographic and genetic consequences of environmental heterogeneity for biological control. Bulletin of Entomological Research 15:237-240.

Lloyd-Smith, J. O., S. J. Schreiber, P. E. Kopp., and W. M. Getz. 2005. Superspreading and the effect of individual variation on disease emergence. Nature 438:355-358.
McArthur, R., and E. O. Wilson. 1967. The theory of island biogeography. Princeton University Press, Princeton, NJ.

McLaughlin, J. F., J. J. Hellman, C. L. Boggs, and P. R. Ehrlich. 2002. Climate change hastens population extinctions. Proceedings of the National Academy of Sciences of the USA 99:6070-6074.

Puerta-Piñero, C., A. Sánchez-Miranda, A. Leverlus, and J. Castro. 2010. Management of burnt wood after fire affects post-dispersal acorn predation. Forest Ecology and Management 260:345-352.

Roslin, T. 2001. Spatial population structure in a patchily distributed beetle. Molecular Ecology 10:823-837.

Roslin, T., and A. Koivunen. 2001. Distribution and abundance of dung beetles in fragmented landscapes. Oecologia (Berlin) 127: 69-77.

Schreiber, S. J., and J. O. Lloyd-Smith. 2009. Invasion dynamics in spatially heterogeneous environments. American Naturalist 174: 490-505.

Smith, A. T, and M. Gilpin. 1997. Spatially correlated dynamics in a pika metapopulation. Pages 407-428 in I. Hanski and M. Gilpin, eds. Metapopulation biology: ecology, genetics and evolution. Academic Press, London.

Snäll, T., J. Ehrlén, and H. Rydin. 2005. Colonization-extinction dynamics of an epiphyte metapopulation in a dynamic landscape. Ecology 86:106-115.

Southwood, T. R. E. 1962. Migration of terrestrial arthropods in relation to habitat. Biological Reviews 37:171-211.

Stelter, C., M. Reich, V. Grimm, and C. Wissel. 1997. Modelling persistence in dynamic landscapes: lessons from a metapopulation of the grasshopper Bryodema tuberculata. Journal of Animal Ecology 66:508-518.

Talley, T. S. 2007. Which spatial heterogeneity framework? consequences for conclusions about patchy population distributions. Ecology 88:1476-1489.

Thomas, C. D. 1994. Extinction, colonization and metapopulations: environmental tracking by rare species. Conservation Biology 8: 373-378.

Tilman, D., R. May, C. Lehman, and M. Nowak. 1994. Habitat destruction and the extinction debt. Nature 371:65-66.

Tilman, P. G., T. D. Northfield, R. F. Mizell, and T. C. Riddle. 2009. Spatiotemporal patterns and dispersal of stink bugs (Heteroptera: Pentatomidae) in peanut-cotton farmscapes. Enviromental Entomology 38:1038-1052.

Vanschoenwinkel, B., S. Gielen, M. Seaman, and L. Brendock. 2008. Any way the wind blows-frequent wind dispersal drives species sorting in ephemeral aquatic communities. Oikos 117:125-134.

Werth, S., H. H. Wagner, F. Gugerli, R. Holderegger, D. Csencsics, J. M. Kalwij, and S. Christoph. 2006. Quantifying dispersal and establishment limitation in a population of an ephiphytic lichen. Ecology 87:2037-2046.

Xu, D., Z. Feng, L. J. S. Allen, and R. K. Swihart. 2006. A spatially structured metapopulation model with patch dynamics. Journal of Theoretical Biology 239:469-481.

Associate Editor: Uta Berger Editor: Judith L. Bronstein 